\title{
Role of Plasticity at Different Sites across the Time Course of Cerebellar Motor Learning
}

\author{
Yan Yang ${ }^{1}$ and Stephen G. Lisberger ${ }^{1,2}$ \\ ${ }^{1}$ Department of Neurobiology and ${ }^{2}$ Howard Hughes Medical Institute, Duke University School of Medicine, Durham, North Carolina 27110
}

Learning comprises multiple components that probably involve cellular and synaptic plasticity at multiple sites. Different neural sites may play their largest roles at different times during behavioral learning. We have used motor learning in smooth pursuit eye movements of monkeys to determine how and when different components of learning occur in a known cerebellar circuit. The earliest learning occurs when one climbing-fiber response to a learning instruction causes simple-spike firing rate of Purkinje cells in the floccular complex of the cerebellum to be depressed transiently at the time of the instruction on the next trial. Trial-over-trial depression and the associated learning in eye movement are forgotten in $<6 \mathrm{~s}$, but facilitate long-term behavioral learning over a time scale of $\sim 5 \mathrm{~min}$. During 100 repetitions of a learning instruction, simple-spike firing rate becomes progressively depressed in Purkinje cells that receive climbingfiber inputs from the instruction. In Purkinje cells that prefer the opposite direction of pursuit and therefore do not receive climbing-fiber inputs related to the instruction, simple-spike responses undergo potentiation, but more weakly and more slowly. Analysis of the relationship between the learned changes in simple-spike firing and learning in eye velocity suggests an orderly progression of plasticity: first on Purkinje cells with complex-spike (CS) responses to the instruction, later on Purkinje cells with CS responses to the opposite direction of instruction, and last in sites outside the cerebellar cortex. Climbing-fiber inputs appear to play a fast and primary, but nonexclusive, role in pursuit learning.

Key words: climbing fibers; floccular complex; long-term depression; smooth pursuit eye movement; synaptic plasticity; trial-over-trial learning

\section{Introduction}

Learning results from "plasticity" that takes the form of changes in the strength of synaptic transmission and/or spike generation in the nervous system. However, plasticity and "learning" are different phenomena. Plasticity is a physiological change in synaptic or cellular function that occurs during learning, but frequently is demonstrated by creating artificial patterns of electrical activity in reduced preparations such as brain slices. Learning defines an adaptive change in behavior and probably results from multiple forms of plasticity at different sites in a neural circuit.

The cerebellum plays an integral role in learning of motor skills. Much is known about cellular mechanisms in the cerebellum (Hansel et al., 2001; Carey, 2011). However, even the successful analysis of the circuit basis for learning in the vestibulo-ocular reflex (Lis-

Received Jan. 3, 2014; revised March 9, 2014; accepted April 9, 2014.

Author contributions: Y.Y. and S.G.L. designed research; Y.Y. performed research; Y.Y. and S.G.L. analyzed data; Y.Y. and S.G.L. wrote the paper.

This work was supported by the Howard Hughes Medical Institute and by the National Eye Institute of the National Institutes of Health (NIH) under award number R01-EY003878. We thank Megan Carey, Court Hull, and members of our laboratory for helpful comments on an earlier version of the manuscript and for helpful discussions. We thank Javier Medina for allowing us to analyze a handful of Purkinje cells from the sample he acquired in our laboratory earlier. K. MacLeod, E. Montgomery, S. Tokiyama, S. Ruffner, D. Kleinhesselink, D. Wolfgang-Kimball, D. Floyd, S. Happel, and K. McGary provided invaluable technical assistance. The content is solely the responsibility of the authors and does not necessarily represent the official views of the NIH.

The authors declare no competing financial interests.

Correspondence should be addressed to Yan Yang, Department of Neurobiology, Duke University School of Medicine, 412 Research Drive, Room 327D, Durham, NC 27710. E-mail: yanyang@neuro.duke.edu.

DOI:10.1523/JNEUROSCI.0017-14.2014

Copyright $\odot 2014$ the authors $\quad 0270-6474 / 14 / 347077-14 \$ 15.00 / 0$ berger, 1994; Blazquez et al., 2004, 2006) showed only snapshots of circuit function before and after learning. Classical conditioning of the eyelid response has yielded insights into possible locations of memory, including how and where timing is learned (Kalmbach et al., 2010; Thompson, 2013). We do not know how and when different plasticity mechanisms are deployed at different sites as learning evolves.

The original theory of cerebellar learning postulated a primary teaching role for the climbing-fiber input to the cerebellum. Sensory inputs over the climbing-fiber system would report errors in a prior movement and would serve as instructions for long-term depression (LTD) at the synapses from active parallel fibers onto Purkinje cells (Albus, 1971; Ito, 1972). LTD would cause semipermanent changes in the simple-spike responses of Purkinje cells, and lead to a gradual improvement in motor performance. Marr (1969) proposed a similar theory based on potentiation of the synapses from parallel fibers to Purkinje cells. Learning in the deep cerebellar nucleus was a later addition to the theory (Miles and Lisberger, 1981).

Observations on the activity of neurons in learning animals are consistent with the cerebellar learning theory (Gilbert and Thach, 1977; Lisberger, 1994; Medina et al., 2000; Christian and Thompson, 2003; Blazquez et al., 2006; Ke et al., 2009; Wulff et al., 2009). But, the cerebellum contains many sites of synaptic and cellular potentiation and depression (D'Angelo et al., 1999; Armano et al., 2000; Hansel et al., 2001; Nelson et al., 2005; Jörntell and Hansel, 2006; Zheng and Raman, 2010; Carey, 2011; Gao et 
al., 2012). Some of the plasticity mechanisms operate in vivo (Jörntell and Ekerot, 2002, 2003; Belmeguenai et al., 2010), but we still need to know which mechanisms operate during behavioral learning. Multiple plasticity mechanisms might operate at different times and sites to support behavioral learning over several time scales (Medina and Mauk, 2000; van Alphen and De Zeeuw, 2002; Blazquez et al., 2004; Boyden et al., 2004).

In the present paper, we analyze learning across different sites and times in pursuit eye movements. Pursuit is an excellent learning system (Kahlon and Lisberger, 1996; Medina et al., 2005; Yang and Lisberger, 2010). The cerebellar floccular complex receives visual climbing-fiber inputs to guide learning (Maekawa and Simpson, 1973; Stone and Lisberger, 1990b). Floccular simple-spike firing controls pursuit (Miles and Fuller, 1975; Lisberger and Fuchs, 1978; Stone and Lisberger, 1990a) and drives behavioral learning (Kahlon and Lisberger, 2000; Medina and Lisberger, 2008). We show now that climbing-fiber inputs play a primary role early in learning, while other cerebellar and brainstem mechanisms appear to contribute later. Our observations elucidate how a temporally and spatially disparate set of cellular and circuit mechanisms cooperate to cause motor learning.

\section{Materials and Methods}

Animal preparation. We report data from experiments on six awake, behaving adult male rhesus monkeys. Four of the monkeys were used at the University of California, San Francisco (UCSF) for recordings from Purkinje cells in the floccular complex during pursuit learning; two of these also provided recordings from Purkinje cells for other papers (Yang and Lisberger, 2013; Yang and Lisberger, 2014) and two provided data used by Medina and Lisberger $(2008,2009)$. Two of the monkeys were used at Duke University for behavioral studies of pursuit learning. Before experiments, we implanted a head holder to prevent head motion during experiments, an eye coil to monitor eye position, and a stainless steel recording cylinder to allow access to the floccular complex for singleneuron recordings (Ramachandran and Lisberger, 2005). The surgical procedures used sterile technique with the monkey under isofluorane anesthesia. Monkeys received opiate and/or nonsteroidal analgesics for several days after each surgery. Procedures were in accordance with the National Institutes of Health Guide for the Care and Use of Laboratory Animals and had been approved in advance by the Institutional Animal Care and Use Committees at UCSF and Duke University.

Behavioral task. Monkeys were trained to fixate and pursue bright spots that were 0.3 or $0.5^{\circ}$ in diameter and appeared on a dark background. We presented visual stimuli on a CRT monitor that was $30 \mathrm{~cm}$ from the monkey's eye and subtended a visual field of $59 \times 47^{\circ}$. The experimental room was lit dimly. After a neuron had been isolated, we presented a baseline block of $\sim 10$ target motions for $850 \mathrm{~ms}$ in each of eight directions at a constant speed of $20 \%$ s. We used the step-ramp trajectory of Rashbass (1961) with a $3^{\circ}$ eccentric step to minimize the occurrence of early saccades during the initiation of pursuit. The data from the baseline block enabled us to assess the preferred direction of the simple-spike responses of the Purkinje cell under study. We used averages of the firing rate and eye movement for target motion in the two directions along the Purkinje cell's preferred axis to quantify the baseline relationship between firing rate and the parameters of eye movement (Medina and Lisberger, 2009).

The main experiment delivered $\geq 2$ learning blocks of 100 or 400 target motions ("trials") using target trajectories and sequences of target motion, which are described below and in Results. Monkeys were rewarded with droplets of fluid at the end of each trial if they kept their eyes within an invisible reward window around the target that was $\pm 1^{\circ}$ during fixation, $\pm 2^{\circ}$ during smooth target motion, and \pm 4 or $5^{\circ}$ after an instructive change in target direction. To avoid punishing the monkey for his inescapable response latencies, fixation requirements were suspended after a change in target position, speed, or direction. There was no explicit reward (or punishment) related to the monkey's performance in the learning conditions.
Data acquisition and analysis. Voltages from a magnetic search coil system provided a measure of eye position and were processed by an analog differentiator to create voltages proportional to horizontal and vertical eye velocity. The differentiator included a filter that rejected signals at frequencies $>25 \mathrm{~Hz}(-20 \mathrm{~dB}$ per decade). We sampled the signals at $1 \mathrm{kHz}$ on each channel and stored them for offline analysis with the spikes from single-unit recordings.

We introduced homemade glass-insulated platinum-iridium microelectrodes daily through the previously implanted cylinder and advanced them into the floccular complex of the cerebellum. Purkinje cells showed occasional complex-spike (CS) responses that interrupted a high level of spontaneous simple-spike firing. We amplified extracellular action potentials conventionally, filtered them with a bandpass of $300 \mathrm{~Hz}$ to $3 \mathrm{kHz}$, and digitized the raw traces at $25 \mathrm{kHz}$ for offline spike sorting. We viewed the spike train for each trial on the computer screen and used a software window discriminator to identify simple spikes and CS responses. We estimated the firing rate for simple spikes with a reciprocal interval algorithm (Lisberger and Pavelko, 1986). We also counted CS responses in bins with a width of $100 \mathrm{~ms}$, and converted the counts to the probability of a CS in each bin.

Most of our data analyses involve averaging firing rate or eye velocity across specific brief time windows, and we detail the analyses and present statistical tests either in the Results or in the relevant figure legends. One more complex data analysis that appears frequently involved measuring how simple-spike firing (or eye velocity) changed between two consecutive learning trials. We call the first and second of each pair of consecutive trials the "instruction" and "test" trials. We sorted the pairs of consecutive trials according to the presence or absence of a CS response to the instructive change in target direction on the instruction trial. Within each group of pairs, we computed the firing rate (or eye velocity) on the test trial minus that on the instruction trial for each millisecond from 350 $\mathrm{ms}$ before to $50 \mathrm{~ms}$ after the time of the instructive change in target direction. We call the values that emerge from this analysis the "trialover-trial change" in neural or behavioral responses. Our goal was to demonstrate and study trial-over-trial changes that are linked to a CS response to the instruction on the first of a pair of trials.

Selection of Purkinje cells for study and experimental design. We studied Purkinje cells in the floccular complex that showed strong modulation of simple-spike firing rate during the smooth pursuit eye movements evoked by "step-ramp" target motions (Stone and Lisberger, 1990a). The eight small peristimulus time histograms in Figure $1 \mathrm{~A}$ summarize the simple-spike direction tuning during pursuit for a typical floccular Purkinje cell. Firing rate showed a strong increase for rightward pursuit, a decrease for leftward pursuit, and very slight increases for upward and downward pursuit. We would say that the simple-spike firing of this Purkinje cell had a rightward "on direction" and a leftward "off direction." Most Purkinje cells in the floccular complex had on directions for simple-spike responses that were toward the side of recording or downward (Krauzlis and Lisberger, 1996).

We customized the learning target motion for each Purkinje cell to match its direction tuning (Fig. 1B, after Medina et al., 2005). In each learning trial, target motion started in a direction orthogonal to the on direction of the Purkinje cell under study at a speed of $20 \%$ s (Fig. $1 C$, downward). The target underwent an "instructive" change in direction $250 \mathrm{~ms}$ after the onset of target motion through the addition of target motion at $30 \%$ in the cell's on direction or off direction for simple-spike responses (Fig. $1 C$, rightward). In the "repeated-direction" paradigm, we presented the same instruction on 100 consecutive trials and induced "long-term" learning. Comparison of the eye velocity in the first and 100th trials (Fig. 1C, blue vs red traces) reveals the expected learned component in horizontal eye velocity (arrowhead) that precedes the instruction, peaks at the time of the instruction, and lasts for $\sim 200 \mathrm{~ms}$ (Medina et al., 2005).

We also used the "random-order" learning paradigm outlined in Figure $1 D$ and described thoroughly by Yang and Lisberger (2010). The learning trials are like those used in the repeated-direction paradigm, but the direction of the instruction is selected randomly on each trial to be in either the on direction or the off direction for the simple-spike responses of the Purkinje cell under study. The random-order learning paradigm 

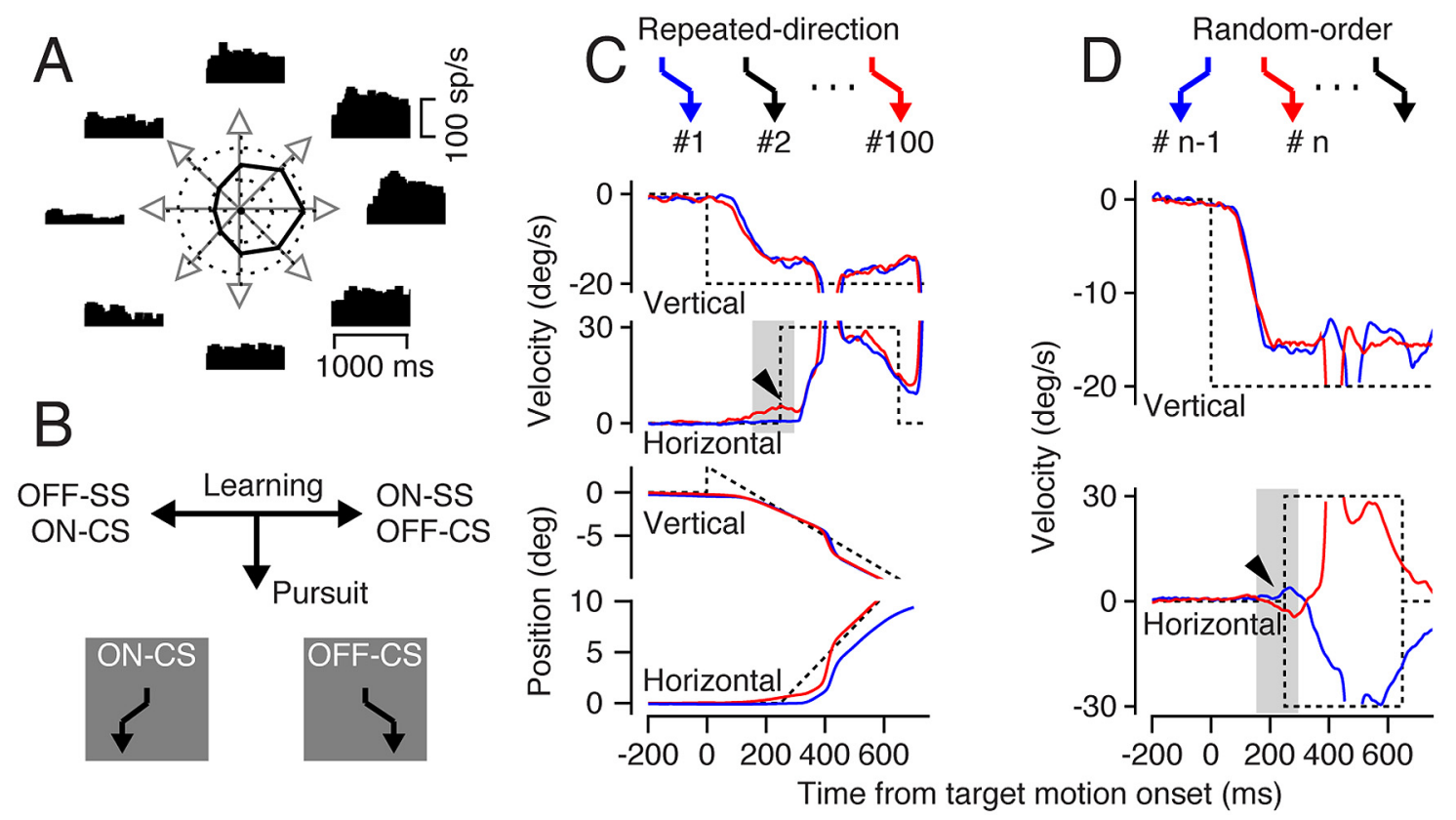

Figure 1. Background information about pursuit learning and the responses of floccular Purkinje cells. $A$, Direction tuning of a Purkinje cell that prefers rightward pursuit. Each peristimulus time histogram shows firing rate as a function of time, and is positioned to represent the direction of pursuit on a polar plot. The black curve is the tuning curve for simple-spike responses. $\boldsymbol{B}$, The arrow labeled "Pursuit" indicates the direction of initial pursuit for an instruction trial. The arrows along the "Learning" axis indicate the directions of the instructive changes in target motion for learning. The zigzags inside the gray squares show the position trajectories for target motion in ON-CS and OFF-CS direction learning trials. $C, \boldsymbol{D}$, The zigzags at the top show sequences of instructive target motions in the repeated-direction $(\boldsymbol{C}$ ) and random-order $(\boldsymbol{D})$ paradigms. $\boldsymbol{C}$, The four pairs of superimposed traces show vertical and horizontal velocity and position as a function of time in example trials. Dashed and continuous traces show target and eye movement. Blue and red traces show responses in the first versus 100 th trial of a repeated-direction learning block. The arrowhead on the horizontal velocity records points out the effect of learning. $\boldsymbol{D}$, The top and bottom pairs of superimposed traces show vertical and horizontal velocity as a function of time in example trials during a random-direction learning block. Dashed and continuous traces show target and eye velocity. Different colored traces show responses in consecutive trials, and are linked to the colors of the zigzags at the top. The arrowhead on the horizontal velocity records points out trial-over-trial learning.

does not induce long-term learning, but causes trial-over-trial learning that can be seen in the examples of Figure $1 D$. At the time of the instruction, eye velocity in the learning direction shows a small deflection in the direction of the instruction on the prior trial. The $n$th trial (red traces) has a small leftward deflection caused by the leftward instruction on the prior, " $n$-first" trial (blue traces). The $n$-first trial has a small rightward deflection caused by the rightward instruction on the $n$-second trial (data not shown). The learned eye velocity precedes both the instruction and the visually guided eye movement caused by the instruction in the current trial. This implies that the responses indicated by the shaded analysis window are related to the instruction in the prior trial, rather than to that on the current trial. In the random-order learning paradigm, trial-overtrial learning is expressed stably across all 400 trials in a random-order learning block, and therefore can be studied quantitatively in a "stationary" state. Randomness in the direction of the instruction discourages anticipation by the monkey, and precludes long-term learning that would change the baseline "state" of the system (Yang and Lisberger, 2010).

\section{Results}

We recorded from Purkinje cells in the floccular complex of the cerebellum because prior research has implicated these Purkinje cells in normal pursuit and pursuit learning (Miles and Fuller, 1975; Lisberger and Fuchs, 1978; Zee et al., 1981; Kahlon and Lisberger, 2000; Rambold et al., 2002; Medina and Lisberger, 2008). We selected Purkinje cells for study if they had strong, directional simple-spike responses during pursuit of step-ramp target motions (Fig. 1A). Almost all responsive Purkinje cells had CS responses with direction selectivity opposite to the simplespike responses (Stone and Lisberger, 1990b; Medina and Lisberger, 2008). Because we are studying effects linked to CS responses, we will refer to Purkinje cells for the rest of the paper according to the direction tuning of their CS responses: learning instructions will be either in the "ON-CS direction" or "OFF-CS direction." Note that the ON-CS direction was the off direction for simple-spike responses, and vice versa (Fig. $1 B$ ). Initially, we discuss the responses of a large fraction of our sample that we call "CS-frequent" Purkinje cells because the probability of an $\mathrm{ON}-\mathrm{CS}$ direction response to a learning instruction was $>3$ times the mean spontaneous probability of 0.09 ( $\pm 0.02 \mathrm{SD})$.

\section{Trial-over-trial neural and behavioral learning are forgotten within $6 \mathrm{~s}$}

We recorded from 19 Purkinje cells during two randomdirection learning blocks of 400 trials each, with "instructiontest" intervals of $2.5 \mathrm{~s}$ between pairs of trials in one block and $6 \mathrm{~s}$ between pairs of trials in the other block. We analyzed pairs of trials where the first trial contained an instructive change in target direction in the ON-CS direction. The next trial served as the test to probe for neural and behavioral learning (schematic at top of Fig. 2). We sorted the pairs according to whether the instruction trial contained a CS in the analysis window from 75 to $175 \mathrm{~ms}$ after an instruction.

We measured how simple-spike firing rate (or eye velocity) changed between the instruction and test trials, and determined how those differences depended on (1) the presence versus absence of a CS response to the instruction and (2) the interval between the instruction and test trials. We computed trial-overtrial changes in firing rate (or eye velocity) for each millisecond from $350 \mathrm{~ms}$ before to $50 \mathrm{~ms}$ after the time of the instructive change in target direction (see Materials and Methods). Our results evaluate learning even though each test trial also contained 

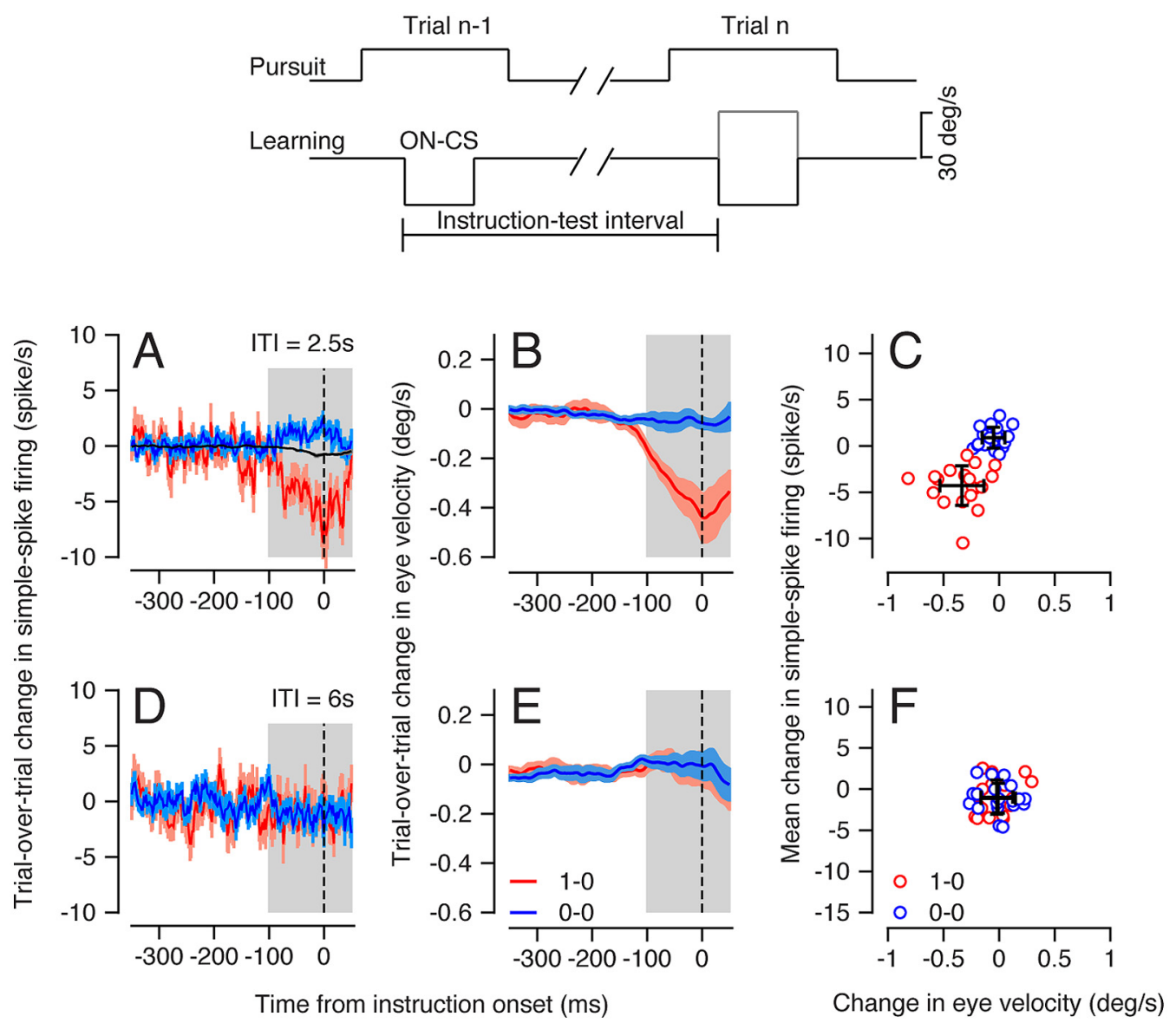

Figure 2. Effect of duration of instruction-test interval on trial-over-trial depression of simple-spike firing rate and trial-over-trial learning in eye velocity. The schematic at the top of the figure shows that target motions used to control the instruction-test interval. Top and bottom traces indicate target velocity in the pursuit and learning directions. $A-C$, Instruction-test interval was $2.5 \mathrm{~s}$. $\boldsymbol{D}-\boldsymbol{F}$, Instruction-test interval was $6 \mathrm{~s}$. $\boldsymbol{A}, \boldsymbol{D}$, Trial-over-trial difference in simple-spike firing rate as a function of time. $\boldsymbol{B}, \boldsymbol{E}$, Trial-over-trial difference in eye velocity as a function of time. Time 0 and the vertical dashed line show the time of the instruction. Red and blue traces show the average across 19 CS-frequent Purkinje cells for $1-0$ versus $0-0$ pairs of successive trials. The ribbon around each trace indicates 1 SEM across the sample of Purkinje cells. The black trace in $A$ indicates the change in simple-spike firing rate predicted by the baseline relationship to eye movement during pursuit before learning. $\boldsymbol{C}, \boldsymbol{F}$, Each symbol shows data for one Purkinje cell. Black crosses show the means and the SDs across Purkinje cells.

an instructive change in target direction: any responses driven by visual inputs would occur $>50 \mathrm{~ms}$ after the instruction, and therefore after the end of the analysis window.

For a $2.5 \mathrm{~s}$ instruction-test interval (Fig. $2 A$ ) but not for a $6 \mathrm{~s}$ instruction-test interval (Fig. 2D), a CS response on the instruction trial was linked to trial-over-trial depression of simple-spike firing rate (red traces) like that reported previously (Medina and Lisberger, 2008; Yang and Lisberger, 2013). Our analysis compares the results on " $1-0$ " pairs where the first trial contained a CS response to the instruction and the second trial did not, and " $0-0$ " pairs where neither trial contained a CS response. For the long instruction-test interval, trial-over-trial depression of simple-spike firing rate was entirely absent for both $1-0$ and $0-0$ pairs of trials (Fig. 2D). For the short interval, trial-over-trial depression of simple-spike firing occurred for 1-0 pairs of trials, but not for 0-0 trials, and reached its nadir at the time of the instruction (Fig. $2 A$, red vs blue traces).

In agreement with a previous paper (Yang and Lisberger, 2013), some trial-over-trial potentiation was visible in the $0-0$ pairs of trials for the short instruction-test interval (Fig. 2A, blue trace). The mean trial-over-trial change in simple-spike firing in the $150 \mathrm{~ms}$ analysis interval (Fig. 2A, gray shading) was not statistically different from that in the prior $150 \mathrm{~ms}$ interval in the 19 neurons studied with short versus long instruction-test intervals
( $p>0.05$ paired $t$ test). However, the small potentiation for $0-0$ trials in Figure $2 \mathrm{~A}$ became statistically significant for a larger group of neurons shown later in the paper. The same potentiation did not appear for $0-0$ pairs of trials for an instruction-test interval of $6 \mathrm{~s}$, perhaps because it is forgotten as quickly as trialover-trial depression.

The trial-over-trial changes in eye velocity paralleled the trialover-trial changes in simple-spike firing, as we have reported before (Yang and Lisberger, 2013). The presence of a CS response on the instruction trial was linked to a trial-over-trial transient change in eye velocity on the test trial when the instruction-test interval was $2.5 \mathrm{~s}$ (Fig. 2C), but not when the instruction-test interval was $6 \mathrm{~s}$ (Fig. $2 F$ ). For the 19 Purkinje cells studied with the $2.5 \mathrm{~s}$ instruction-test intervals in the present dataset, trialover-trial learning in eye velocity did not appear if the Purkinje cell under study did not emit a CS in response to the change in target direction on the instruction trial. For other samples of Purkinje cells, trial-over-trial learning in eye velocity was present but much smaller for $0-0$ versus $1-0$ pairs (Yang and Lisberger, 2013). As we have noted before (Medina and Lisberger, 2008; Yang and Lisberger, 2013), the difference in the trial-over-trial learned eye velocity between $1-0$ and $0-0$ pairs implies that the presence and absence of CS responses tends to be correlated across the Purkinje cell population. If CS responses occurred with 
independent probabilities in each Purkinje cell, then the fluctuations across the population in trial-over-trial depression would average out; the trial-over-trial learned eye velocity would be the same for $1-0$ and $0-0$ pairs.

Figure $2 A, B, D, E$ shows mean and SEs as a function of time across recordings from the 19 Purkinje cells we studied with both instruction-test intervals. Figure $2 C, F$ plots summary data from each neuron as a separate point to illustrate the presence of the same effects in individual Purkinje cells. The red and blue symbols were statistically different for the $2.5 \mathrm{~s}$ instructiontest interval in Figure $2 C$ (paired $t$ test; $n=19, p<0.001$ ), but not for the $6 \mathrm{~s}$ instruction-test interval in Figure $2 F$ (paired $t$ test; $n=19, p>0.5$ ).

Next, we tested whether the absence of trial-over-trial depression or learning for longer intervals reflects a failure to learn versus failure to remember. We took advantage of the fact that 2.5 and $6 \mathrm{~s}$ instruction-test intervals actually alternated in the same learning block when we tested trial-over-trial depression and learning with a $6 \mathrm{~s}$ interval. This allowed us to analyze data for both instruction-test intervals in the same stream of data. The pairs with short intervals served as a control to validate that the system was in a learning mode, while the pairs with intervals of $6 \mathrm{~s}$ evaluated how much learning was remembered after $6 \mathrm{~s}$. The probability of a CS response to the instruction was almost identical for the two instruction-test intervals (schematic at top of Fig. 3). However, trial-over-trial depression of simple-spike firing was present for $2.5 \mathrm{~s}$ instruction-test intervals and absent for 6 s intervals (Fig. 3C). Trial-over-trial learning in eye velocity also was forgotten after a $6 \mathrm{~s}$ instruction-test interval (Fig. 3D). The effect of instruction-test interval was statistically significant in Figure $3 C, D(p<0.01$, paired, two-sided Wilcoxon signed rank test). We conclude that CS-linked trial-over-trial effects on both neural and behavioral learning are forgotten in $<6 \mathrm{~s}$.

Maruta et al. (2007) discovered that CS responses often occurred more frequently than the average of $\sim 1 \mathrm{CS} / \mathrm{s}$. In our data for an instruction-test interval of $2.5 \mathrm{~s}, 8.5 \%$ of learning trials contained $>1$ CS response during the $400 \mathrm{~ms}$ instruction. The magnitude of trial-over-trial depression of simple-spike responses, however, did not depend on whether an instruction caused one versus two CS responses.

\section{Relationship between trial-over-trial learning and learning over a longer time course}

Next, we asked whether long-term learning would be compromised under conditions when trial-over-trial effects were forgotten between instructive stimuli. We used the "repeated-direction paradigm" with the same direction of instruction and same instruction-test interval in all 100 trials of a learning block (schematic at top of Fig. 4A). Different learning blocks on a given experimental day presented different instruction-test intervals $(2.5,6$, or $10 \mathrm{~s})$ and/or different directions of instructive target motion. We measured the "learned eye velocity" as the mean across the interval from $100 \mathrm{~ms}$ before to $50 \mathrm{~ms}$ after the time of the instruction (Fig. $4 \mathrm{~A}$, gray shading on eye velocity traces). This interval covers the time when learning is expressed, and ends before any visually guided response to the instruction on that trial.

The duration of the instruction-test interval had a large effect on the magnitude of long-term learning during the repeateddirection paradigm. For upward instructions (Fig. 4B), learning was twice as large over the entire course of the learning block when the instruction-test interval was $2.5 \mathrm{~s}$ (red) versus 6 (blue) or $10 \mathrm{~s}$ (yellow). The same was true for rightward and leftward
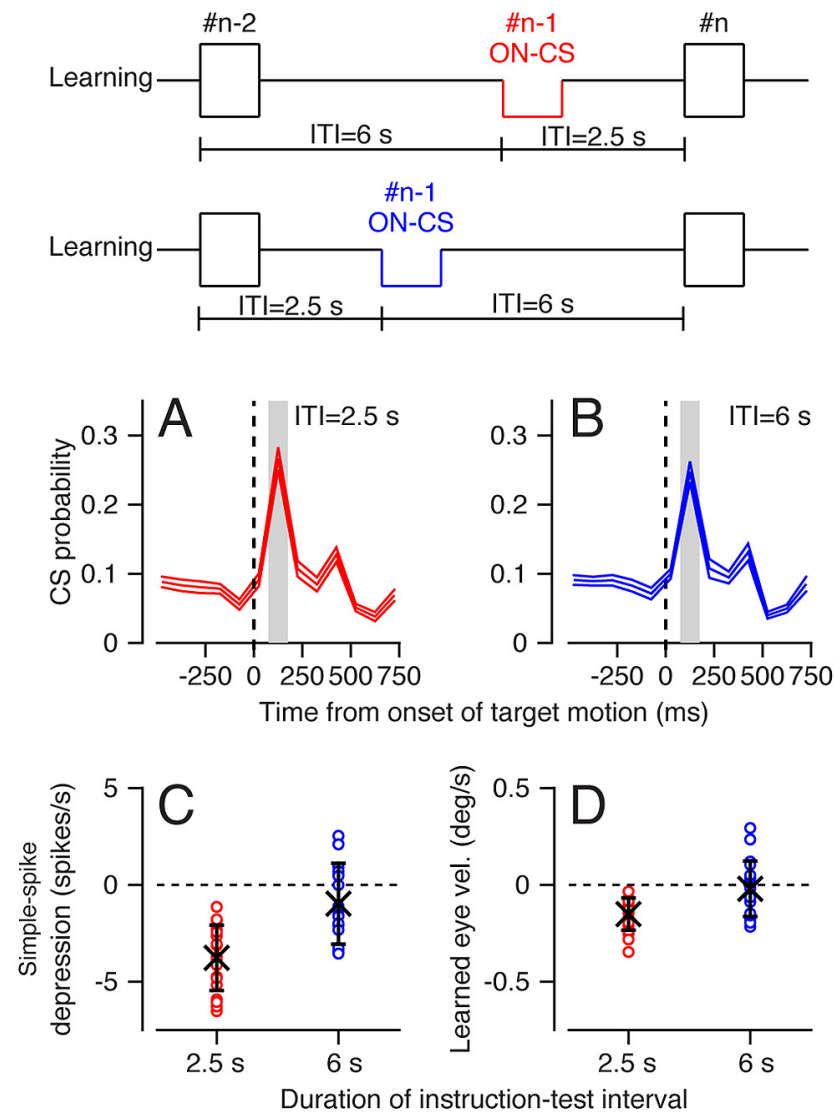

Figure 3. Use of interleaved long and short instruction-test intervals to demonstrate forgetting in trial-over-trial depression and learning. Top panel shows schematic of target motions used to interleave instruction-test intervals of 2.5 and $6 \mathrm{~s}$. Trial $\# n-1$ is the instruction trial, and trial $\# n$ is the test trial. $A, B, C S$ response probability as a function of time averaged across the 19 Purkinje cells. Vertical dashed lines show the time of the instruction. Gray shading indicates the analysis interval for CS probability. C, D, Comparison of trial-over-trial depression of simplespike firing rate $(\boldsymbol{C})$ and trial-over-trial learning in eye velocity $(\boldsymbol{D})$ for different instruction-test intervals. Each symbol shows data for recordings from a single Purkinje cell. The black X's and error bars show the mean \pm 1 SD across the population of 19 CS-frequent Purkinje cells. Red and blue traces $(\boldsymbol{A}, \boldsymbol{B})$ and symbols $(\boldsymbol{C}, \boldsymbol{D})$ indicate instruction-test intervals of 2.5 versus $6 \mathrm{~s}$.

learning (data not shown). For downward instructions, in contrast, the learning curves did not vary as a function of instruction-test interval (Fig. 4C). We quantified the effect of instruction-test interval and instruction direction by averaging the final magnitude of learning across the last 10 trials in each learning block, and across 10 learning blocks for each direction of instruction in each monkey.

When we considered learning for instructions in all four cardinal directions, the asymptotic magnitude of learning was larger for an instruction-test interval of 2.5 versus $6 \mathrm{~s}$, and was direction dependent (Fig. $4 D, E$, red vs blue symbols). The difference between the means for instruction-test intervals of 2.5 versus $6 \mathrm{~s}$ was statistically significant for upward, leftward, and rightward target motion in both monkeys ( $p<0.01$, two-tailed $t$ test), but not for downward target motion in either monkey $(p>0.1)$. As expected from inspection of the graphs in Figure $4 D, E$, ANOVA yielded significant effects of both direction and instruction-test interval, with an interaction term that was statistically significant.

Figure 4 suggests that long-term learning in pursuit is facilitated by a neural correlate of CS-linked trial-over-trial learning. The directional organization of the simple-spike and CS responses of floccular Purkinje cells (Stone and Lisberger, 1990b; 
Krauzlis and Lisberger, 1996) suggests that trial-over-trial depression in the floccular complex is a strong candidate for a neural event that might facilitate longterm learning. In each floccular complex, CS responses for vertical learning directions will be evoked by upward instructions in Purkinje cells that prefer pursuit along the vertical axis. Purkinje cells on both sides of the cerebellum will exhibit trial-over-trial depression of simple-spike firing rate. The two floccular complexes will cooperate to create a large learned upward eye movement. Trial-over-trial effects could facilitate long-term learning strongly on both sides of the cerebellum. For Purkinje cells that prefer horizontal pursuit, CS responses will be evoked by rightward or leftward target motion in the left or right floccular complex, respectively. Trial-over-trial depression will occur only in one floccular complex, longterm learning would be facilitated in one floccular complex at a time, and each side would work individually to create a learned eye movement of moderate amplitude.

The absence of a large population of Purkinje cells with CS responses for downward instructions (Stone and Lisberger, 1990b; Krauzlis and Lisberger, 1996) could explain the asymmetry between upward and downward trial-overtrial learning (Yang and Lisberger, 2010).

Downward instructions will cause very little CS-linked trial-overtrial simple-spike depression in the floccular complex. The absence of downward trial-over-trial plasticity may deprive the system of the facilitation provided by trial-over-trial learning, and could explain (1) the almost identical downward learning curves for instruction-test intervals of 2.5 and $6 \mathrm{~s}$ (Fig. 4C) and (2) the larger upward long-term learning for the short instruction-test interval. We conclude that CS responses may play a primary role in the neural changes that lead to long-term learning, and that the floccular complex may be the site where plasticity occurs earliest during pursuit learning.

\section{Direction-dependent learning curves for simple-spike firing rate}

The previous section suggests that CS-linked plasticity facilitates long-term learning over a time course of 100 trials. This tentative conclusion implies that we should compare the neural learning curves over the same time course for instructions in the ON-CS versus OFF-CS directions. From first principles, the presence of CS responses to the instruction should cause depression of simple-spike firing rate while the absence should cause potentiation of simple-spike firing rate. Given that separate cellular mechanisms may be involved, we might see a difference in the time course or magnitude of depression versus potentiation in simple-spike firing rate, depending on the presence or absence of CS responses to the instructions.

Figure 5 validates the prediction of different time courses of neural learning for instructions in the ON-CS versus OFF-CS directions. To obtain the curves in Figure 5, we divided the 100
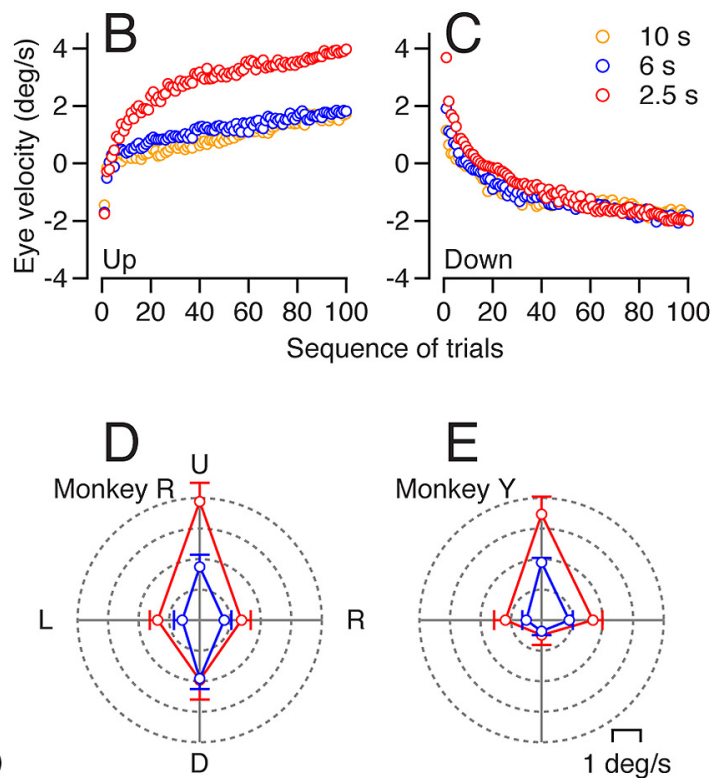

$1 \mathrm{deg} / \mathrm{s}$

Effect of instruction-test interval on longer-term learning in pursuit. $\boldsymbol{A}$, Zigzags at the top show the learning target (n) same instruction for 100 consecutive trials with an upward $(\boldsymbol{B})$ or downward $(\boldsymbol{C})$ direction, and each block began with what appears to be very rapid learning but really is rapid recovery from prior learning. Thus, Trial \#2 would be a better index of the eye velocity at the start of a block.

trials in a repeated-direction learning block into sets of 10 trials. We then computed how the learned component of the simple-spike response evolved from set to set. We used the method outlined in Figure $5 \mathrm{~A}$ to isolate the learned component of the simple-spike response from the small increase in simple-spike firing rate associated with the initiation of pursuit in the learning trials. We superimposed the average firing rates for target motion without an instruction before the learning block and for data obtained during learning (Fig. 5A, top, red and black traces). We computed the "learned" change in simple-spike firing rate as the difference between these two traces at each millisecond of the traces (Fig. $5 A$, bottom, red trace). We then reported averages across the analysis interval (gray shading).

For Purkinje cells that preferred horizontal eye motion, learned depression of the simple-spike responses of floccular Purkinje cells evolved more quickly when the instruction was in the ON-CS direction (Fig. 5B, red symbols), compared with the potentiation when the instruction was in the OFF-CS direction (blue symbols). The pink ribbons assist in direct comparison of the time courses of learned depression and potentiation in simple-spike firing rate. The ribbons show the absolute values (in the algebraic sense) of the red symbols in the same figure. They provide visual documentation that the depression appears more quickly in the early part of the learning block. Yet, potentiation catches up so that the changes in simple-spike firing rate are symmetrical by the end of a repeated-direction learning block of 100 trials. The time courses of horizontal eye velocity learning are almost identical throughout the learning block for the ON-CS 

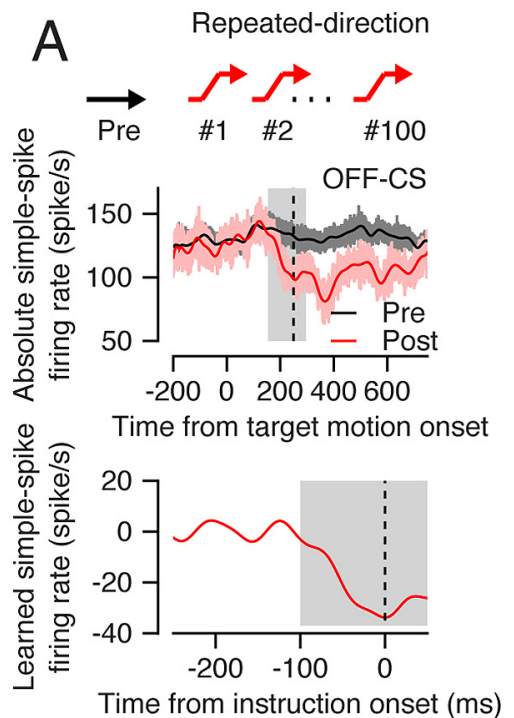
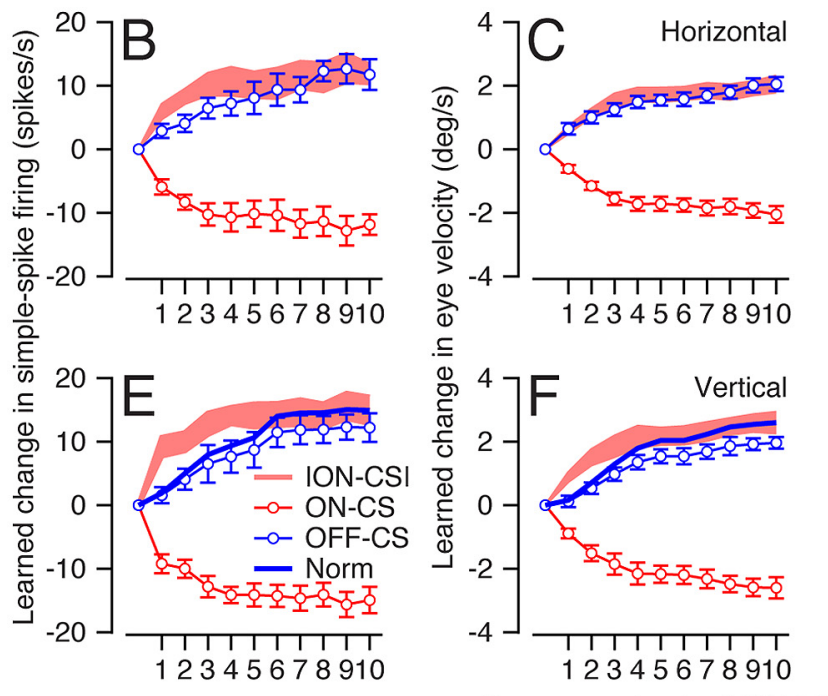

Sequence of sets of 10 trials

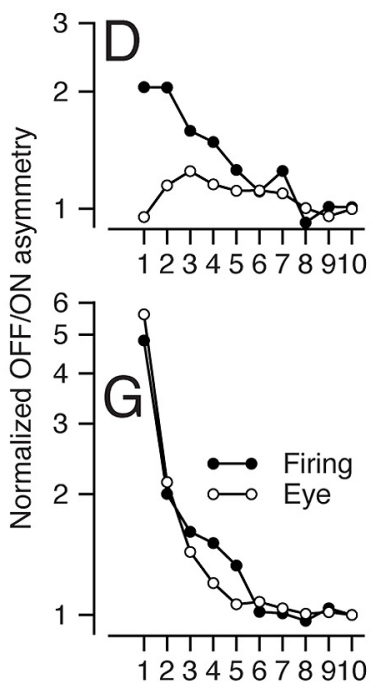

Figure 5. Differences in time course of neural learning for instructions that did versus did not evoke CS responses. $A$, Top, Black arrow shows the prelearning control target motion and the red zigzags show the target motion in learning trials. In the upper graph, the black and red traces and error ribbons show the time course of absolute simple-spike firing rate averaged across the prelearning trials and the last 10 learning trials. In the lower graph, the red trace of learned simple-spike firing rate shows the millisecond-by-millisecond difference between the firing rate in the last 10 learning trials and the prelearning control. Gray shading shows the analysis interval for the graphs in the rest of the figure, and the vertical dashed line shows the time of the instruction. $\boldsymbol{B}-\boldsymbol{G}$, Top versus bottom rows of graphs show data for Purkinje cells that preferred horizontal versus vertical eye motion. $\boldsymbol{B}, \boldsymbol{E}$, Learning curves for simple-spike firing rate. $\boldsymbol{C}, \boldsymbol{F}$, Learning curves for eye velocity. Blue and red symbols show data for learning in the OFF-CS direction versus ON-CS direction for the Purkinje cell under study. $\boldsymbol{B}, \boldsymbol{C}, \boldsymbol{E}, \boldsymbol{F}$, Pink ribbons show the red traces inverted to allow direct comparison with the blue traces. Bold blue traces without symbols in $\boldsymbol{E}$ and $\boldsymbol{F}$ show the data for the $0 \mathrm{~N}$ - $\mathrm{CS}$ direction scaled to equal the inverse of the OFF-CS direction in the 10th set of 10 trials. Error bars show SEMs across 19 and 18 CS-frequent Purkinje cells that preferred horizontal and vertical pursuit. D, G, Asymmetry between OFF-CS and ON-CS directions, normalized to be equal to 1 in the 10 th set of 10 trials. Filled and open symbols show data for learned changes in simple-spike firing rate and eye velocity.

and OFF-CS directions (Fig. 5C, compare red ribbons, blue symbols). We quantified the asymmetries by calculating the ratio of the magnitudes of the learned changes of simple-spike firing rate and the eye velocity in the two directions: $|O N-C S| / O F F-C S$. The asymmetry in firing rate (Fig. $5 D$, filled symbols) was twofold in the first set of 10 trials, and declined over the first 40 trials until it was close to 1 . The asymmetry in eye velocity (Fig. $5 D$, open symbols) was close to 1 throughout the learning curves.

For Purkinje cells that preferred vertical eye motion (Fig. 5, bottom row of graphs), the conclusions are the same. However, the asymmetry in eye velocity learning for upward versus downward instructions (Figs. $4 D, E, 5 F$ ) rendered the data more complicated. Comparison of the learning curves for the OFF-CS direction (Fig. $5 E, F$, blue symbols) with the absolute value of the data for the ON-CS direction (red symbols and pink ribbon) shows asymmetries in the time course of the learned changes in both simple-spike firing rate and eye velocity. The data also reveal an asymmetry in the amount of asymptotic learning in the 10th set of 10 trials for both neural activity and behavior. To assess the time course of the asymmetries, we first eliminated the magnitude asymmetry in the 10th set of 10 trials. We normalized the data for the OFF-CS direction by scaling to match the amplitude of the data for the ON-CS direction (blue traces without symbols). Then, we computed the ratio: $\mid O N$ $C S \mid /(A \times O F F-C S)$, where $A$ is the scaling factor used for normalization. Now, both simple-spike firing rate and eye velocity show strong and nearly equal asymmetries in the first two sets of 10 trials; the firing rate shows a larger asymmetry in the third through fifth sets of 10 trials; and the asymmetries match after the fifth set of 10 trials (Fig. $5 G)$.

We suggest that the organization of preferred directions for the population of floccular Purkinje cells (Krauzlis and Lisberger, 1996) can explain the time course and magnitudes of the neural and behavioral learning curves for Purkinje cells that prefer horizontal versus vertical eye motion.

We further suggest that behavioral learning in horizontal pursuit is symmetrical because of the reciprocal organization of the Purkinje cells in the floccular complexes on the two sides of the cerebellum. We think that the asymmetry of the time courses of neural learning for the ON-CS and OFF-CS directions reflects simple-spike depression that occurs more quickly than does potentiation.

Finally, we suggest that learning in vertical pursuit shows an up-down asymmetry throughout 100 learning trials because of the absence of Purkinje cells with ON-CS directions that are downward. The asymmetry is most pronounced early in a repeated-direction learning block because of the absence of CS-linked trial-over-trial depression for downward instructions. We suggest that the residual asymmetry in simple-spike learning after normalization again reflects quicker simplespike depression versus potentiation.

\section{Learned simple-spike firing rate exaggerates the magnitude of} learned eye velocity

The learned changes in the simple-spike firing rate of floccular Purkinje cells might result from plasticity that occurs outside the cerebellum and that is transmitted to the cerebellum over mossy fiber afferents. For example, the floccular complex receives feedback from the brainstem about the ongoing eye movement (Lisberger and Fuchs, 1978; Stone and Lisberger, 1990a; Lisberger, 2009). If the changes in simple-spike firing are inherited from plasticity in precerebellar pathways, however, we might predict that the magnitude of the learned changes would agree with the magnitude of what we would call the "baseline" response during control pursuit before learning. It follows that changes in Purkinje cell 
simple-spike firing rate must be larger than those expected from feedback to provide evidence that plasticity in the cerebellum drives behavioral learning.

Our next step was to compare the actual learning curves for simple-spike firing rate with those expected from feedback about the millisecond-by-millisecond time course of the learned eye velocity. We define the change in simplespike firing rate expected from feedback according to what we call the "baseline" relationship between firing rate and the parameters of eye movement during prelearning smooth pursuit eye movements (Medina and Lisberger, 2009). In the floccular complex, the baseline relationship between firing rate and eye movement during pursuit of step-ramp target motion is defined by the following regression equation (Eq. 1):

$$
f r=r r+k E+r \dot{E}+a \ddot{E}
$$

where $E, \dot{E}$, and $\ddot{E}$ represent eye position, velocity, and acceleration; $r r$ is the spontaneous firing rate during fixation at straight-ahead gaze; and $k, r$, and $a$ are regression coefficients that are unique to each Purkinje cell. Equation 1 does an excellent job of reproducing the trajectory of mean simplespike firing rate during pursuit of step-ramp target motions (Medina and Lisberger, 2009).

For each Purkinje cell, we computed the regression coefficients for Equation 1 separately for the OFF-CS and ON-CS directions of pursuit of step-ramp target motions. Inspection of the traces revealed that the regression model fit the data well for all neurons, except for noise in firing rate due to averaging across only $\sim 10$ target/eye movements. We then applied Equation 1 to the time-varying trajectory of the learned eye velocity to predict simple-spike firing rate as a function of time for each learning trial. Finally, we quantified the predicted firing rate in exactly the same way as we had quantified the actual firing rates, by averaging across the analysis interval and across groups of 10 trials. One sample for this analysis came from the data used in the rest of the present paper $(n=28)$, and one was mined from the dataset for a prior paper (Medina and Lisberger, 2008; $n=9$ ). We had a larger number of Purkinje cells in the present sample because our experimental design depended on excellent isolation of CS responses; we had made CS isolation a priority during data collection. All 37 recordings satisfied the criteria for "CSfrequent" Purkinje cells.

For learning in the ON-CS direction, the actual depression of simple-spike firing rate (Fig. 6A, $C$, red symbols) exceeded by 2.5 -fold the depression predicted from the baseline relationship between firing rate and eye movement (black symbols). The difference was present in the first set of 10 learning trials, and for both horizontal (Fig. 6A) and vertical (Fig. 6C) pursuit. The actual change in firing rate was 2.6 and 2.4 times the predicted change after 20 and 100 learning trials. For learning in the OFF-CS direction, the actual potentiation of simple-spike firing rate was smaller: only 1.5 and 1.7 times the predicted change after 20 and 100 learning trials.
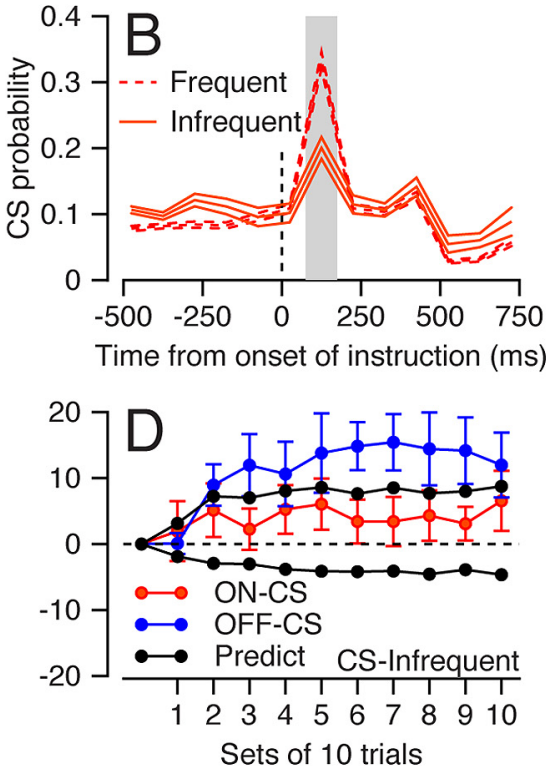

Sets of 10 trials

Horizontal<smiles>[Y10]C1CCCC1</smiles>

$-2$

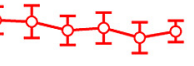

ON-CS

Predict

Vertical
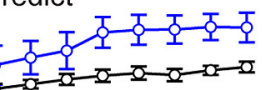

$a \rightarrow a-a-a-a-a$

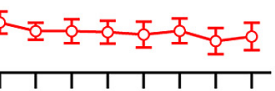

910

Sets of 10 trials

Figure 6. Neural learning curves in the cerebellum for Purkinje cells with frequent versus infrequent CS responses. $A, C$, Data for ( $n=10) \cdot A, C, D$, Blue and red symbols show the progression of simple-spike learning for the OFF-CS direction versus $0 N-C S$ determined solely by the baseline relationship between firing rate and the parameters of eye movement during pursuit of stepramp target. $\boldsymbol{B}$, CS probability as a function of time. Dashed and continuous traces show data for CS-frequent and CS-infrequent Purkinje cells. In all panels, error bars and bands show SEMs across neurons.

The CS-linked trial-over-trial depression of simple-spike firing rate documented in the random-direction learning paradigm also exaggerated the behavioral learning. For the 19 Purkinje cells that were studied in the random-direction learning paradigm in Figure $2 B$, the parameters from the regression analysis predict a trial-over-trial change in simple-spike firing rate (Fig. $2 B$, black trace) that is tiny compared with the actual trial-over-trial depression (red trace). The actual CS-linked depression of simplespike firing was almost 10 times that predicted from the baseline relationship to eye movement.

A simple algebraic model explains why we might find that the neural expression of learning exaggerates the behavioral learning. Assume that the signals that drive baseline pursuit before learning can be represented as having three sources. Smooth eye velocity will be defined by the sum of the signals that emanate from the three pathways as expressed in the following equation (Eq. 2):

$$
\Delta E=\Delta P C_{O N-C S}+\Delta P C_{O F F-C S}+\Delta B S .
$$

If equal changes in firing rate in all three pathways support the eye movement during baseline pursuit (i.e., $\triangle P C_{O N-C S} \equiv$ $\triangle P C_{\text {OFF-CS }} \equiv \triangle B S$ ), then Equation 2 reduces to $\triangle E=3 \cdot \Delta P C_{\text {ON-CS }}$. The baseline "sensitivity" to eye velocity in the Purkinje cells represented by $P C_{O N-C S}$ will be $\Delta P C_{O N-C S} / \Delta E=0.33$. Suppose that a learned change in eye movement is caused by changes in firing rate only in $P C_{O N-C S}$. Then, $\Delta E=\Delta P C_{O N-C S}$ and the sensitivity to eye velocity will be $\Delta P C_{O N-C S} / \Delta E=1.0$. Learned changes in firing rate in only one of three pathways in the pursuit circuit would predict that the sensitivity to learned eye velocity in that pathway would be three times the baseline sensitivity. The logic of Equation 2 is simplest under our assumption of equal contributions from each of the three sites in the model, but the general conclusions hold if there are more sites or if the contributions are unequal. Also, our earlier analysis of learning in the vestibulo-ocular reflex substan- 
tiated the logic of Equation 2. One group of brainstem interneurons in the vestibulo-ocular reflex pathways showed changes in firing rate that far exaggerated the changes predicted by the learned eye movement behavior (Lisberger et al., 1994). Those interneurons almost certainly are one site of plasticity that drives motor learning (Lisberger et al., 1994; Blazquez et al., 2006).

In the context of Equation 2, our data show that sensitivity is much higher in Purkinje cells for learned changes in eye velocity than for normal, baseline pursuit (Fig. 6A,C). For instruction directions that cause a high probability of CS responses, learned simple-spike firing rate far exaggerates the learned eye movement on the first learning trial, and remains $\sim 2.5$ times larger than predicted on the 100th trial. For instruction directions that inhibit CS responses, learned potentiation of simple-spike firing rate is quite small on the first learning trial, and increases up to a level that is $\sim 1.5$ times larger than predicted on the 100th trial. We suggest that Purkinje cells are primary sites of learning and that other parts of the pursuit circuit become engaged in learning later. We take the differences in time course and amplitude of depression and potentiation as evidence for separate mechanisms that have different time courses and that do versus do not depend on CS responses.

\section{CS-frequent versus CS-infrequent Purkinje cells}

Up to this point in the paper, we have analyzed only Purkinje cells that we call "CS-frequent" because they showed CS responses to the instruction with a probability $>3$ times the spontaneous probability (Fig. 6B, dashed traces). A smaller, second group of "CS-infrequent" Purkinje cells showed a much lower probability of CS responses to the instruction (Fig. $6 B$, continuous traces). We introduce the latter group of Purkinje cells now.

As documented in a previous study (Medina and Lisberger, 2008), CS-infrequent Purkinje cells showed "wrong-way" learning in simple-spike firing rate during 100 trials of learning in the ON-CS direction. Even though CS responses to the instruction occurred on $\sim 20 \%$ of the learning trials, simple-spike firing rate was progressively potentiated, rather than depressed (Fig. $6 D$, red symbols). The wrong-way simple-spike learning for the ON-CS direction in CS-infrequent Purkinje cells cannot be attributed to the baseline response properties during pursuit for CSinfrequent Purkinje cells (Fig. 6D, black symbols). Indeed, CSfrequent and CS-infrequent Purkinje cells had similar responses during baseline pursuit in our data. The same was true for the Group 1 versus Group 2 Purkinje cells of Medina and Lisberger (2008). In the OFF-CS direction, simple-spike learning of the CS-infrequent Purkinje cells (Fig. 6D, blue symbols) was very similar to that for the CS-frequent Purkinje cells, and again exaggerated the behavioral learning.

The differences between CS-frequent and CS-infrequent Purkinje cells also appeared in the analysis of learning in individual neurons (Fig. 7). Here, symbols for learning in the ON-CS direction (Fig. 7A) would plot to the left of the oblique line if the actual simple-spike learning exaggerated the learned change in behavior. Symbols would plot to the right of the oblique line if the amount of simple-spike learning was less than predicted by the baseline pursuit response. In our samples, 8 of 10 CS-infrequent Purkinje cells (filled symbols) but none of the CS-frequent Purkinje cells (open symbols) plotted to the right of the oblique line. Thus, we conclude that CS-frequent and CS-infrequent Purkinje cells show different signs of learned changes in simple-spike firing during 100 trials of learning, at least in the ON-CS direction. The learning of the CS-infrequent Purkinje cells in the ON-CS direction adds to the evidence that learned depression of simple-

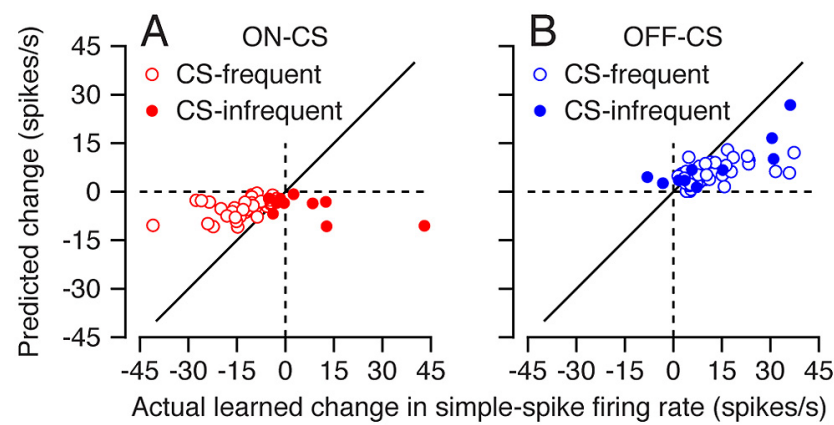

Figure 7. Cell-by-cell analysis of whether learned changes in simple-spike firing rate exaggerate the behavioral changes in eye velocity. Each symbol shows results from an individual Purkinje cell. Filled and open symbols show results for CS-infrequent versus CS-frequent Purkinje cells. $\boldsymbol{A}$, Changes in simple-spike firing rate exaggerate behavioral learning if the symbol plots to the left of the oblique line. $\boldsymbol{B}$, Changes in simple-spike firing rate exaggerate behavioral learning if the symbol plots to the right of the oblique line.

spike firing rate requires abundant CS responses during the learning block.

For the OFF-CS direction (Fig. 7B), CS-frequent and CSinfrequent Purkinje cells showed more similar learning. Here, symbols plotting to the right versus left of the oblique line would exaggerate versus underestimate the learned change in behavior. In our data, 6 of 10 CS-infrequent Purkinje cells and 32 of 37 CS-frequent Purkinje cells plotted to the right of the oblique line and therefore show simple-spike learning that exaggerates the behavioral learning.

We realize that CS-frequent and CS-infrequent Purkinje cells may actually be two ends of a continuum, and we chose to divide them into two groups for simplicity of analysis. Our results agree completely with those from Medina and Lisberger (2008), even though they divided the Purkinje cells into two groups according to the direction of the learned change in simple-spike firing rate whereas we divided them according to the CS probability. These two features are tightly related. Because we suspect that the CS probability is causal, we prefer to use that measure to divide the neurons into groups.

\section{Basis for different learning in CS-frequent versus CS- infrequent Purkinje cells}

Why do CS-frequent versus CS-infrequent Purkinje cells show learned changes with opposite signs during repetition of ON-CS direction learning trials? One possibility is that the differences in the density of climbing-fiber input to the two groups determine the differences in simple-spike learning. For example, the low probability of CS responses during ON-CS direction learning may allow synaptic potentiation to occur on $>80 \%$ of the trials, and to dominate the small amount of synaptic depression created by the paucity of CS responses to the instruction. A second possibility is that the CS-infrequent Purkinje cells lack CS-linked trial-over-trial depression of simple-spike responses.

CS-frequent and CS-infrequent Purkinje cells both show CSlinked trial-over-trial plasticity of simple-spike firing. In both groups of neurons (Fig. $8 A, B$ ), the trial-over-trial change in simple-spike firing rate shows a depression timed to the instruction when the instruction trial contains a CS response to the instruction (red traces). The trial-over-trial change in simple-spike firing rate is nearly flat when the instruction trial lacks a CS response to the instruction (blue traces). The data are somewhat noisier for the CSinfrequent Purkinje cells partly because of small neuron numbers and partly because of smaller numbers of CS responses. 

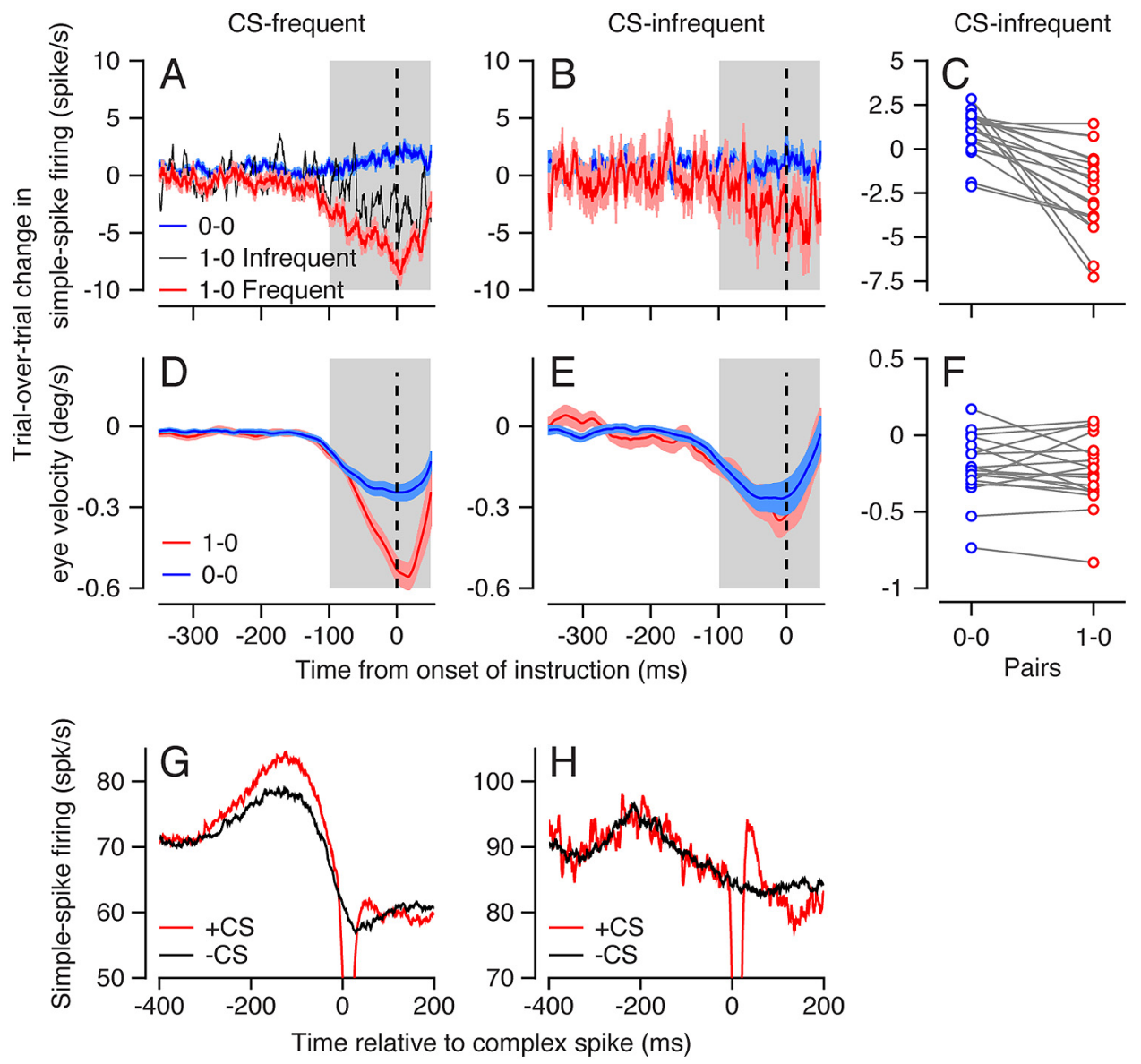

Figure 8. Comparison of CS-linked trial-over-trial plasticity and learning in CS-frequent and CS-infrequent Purkinje cells. Left column shows data for CS-frequent Purkinje cells; middle and right columns show data for CS-infrequent Purkinjecells. $A, B$, Each trace shows the average trial-over-trial change in firing rate as a function of time relative to the instruction onset. Black trace in $A$ is the mean from the red trace for $C S$-infrequent Purkinje cells in $\boldsymbol{B} . \boldsymbol{D}, \boldsymbol{E}$, Each trace shows the trial-over-trial change in eye velocity as a function of time relative to the instruction onset. $\boldsymbol{A}, \boldsymbol{B}, \boldsymbol{D}, \boldsymbol{E}$, Pale ribbons indicate 1 SEM across Purkinje cells, the vertical dashed line shows the time of theinstruction, and the gray shaded area indicates the analysis intenval. $\boldsymbol{C}, \boldsymbol{F}$, Each symbol plots themean trial-over-trial changein simple-spikefiring rate ( $\boldsymbol{C}$ or eyevelocity $(\boldsymbol{F})$ for an individual Purkinjecell, with symbols from the same cell connected by gray lines. $A-\boldsymbol{F}$, Red and blue traces/symbols show data for $1-0$ versus $0-0$ pairs of trials. $\mathbf{G}, \boldsymbol{H}$, Absolute simple-spikefiring rate as a function of time relative to the occurrence of a $C S$ response. Red and black traces show averages for trials with and without a $C S$ response. $I n$ " $-C S^{\prime \prime}$ trials, we aligned the traces on the time of occurrence of the CS responseto the instruction on the prior " $+C S^{\prime \prime}$ trial. The traces in $A$ and $D$ are reproduced from our prior paper (Yang and Lisberger, 2013) under the terms of the Creative Commons Attribution License.

CS-linked trial-over-trial depression of simple-spike firing is genuinely somewhat smaller in the 18 CS-infrequent versus the 90 CS-frequent Purkinje cells (Fig. 8A, compare red, black traces). We can ascribe the difference to the presence versus absence of "same-trial facilitation" of simple-spike firing. In the CS-frequent, but not the CS-infrequent, Purkinje cells simplespike firing rate is higher on instruction trials that have a CS response (Fig. $8 G, H$ ). The presence of same-trial facilitation on the instruction trial but not the test trial in CS-frequent Purkinje cells inflates the trial-over-trial depression of simple-spike firing. In a previous paper, we provided evidence that same-trial facilitation reflects the degree of control of inferior olive firing by the disynaptic pathway from Purkinje cells to the inferior olive (Yang and Lisberger, 2013).

The CS-linked trial-over-trial learning of eye velocity is quite different between the CS-frequent and the CSinfrequent Purkinje cells. For the CS-frequent Purkinje cells, the trial-over-trial learning in eye velocity is linked to whether or not a CS response occurred on the instruction trial (Fig. $8 D$ ). Learning is large when the instruction evokes a CS response, and quite small when it does not. We have argued before for the CS-frequent Purkinje cells that the link from the presence of a CS on the instruction trial to much larger trial- over-trial learning in the behavior is caused by correlations in the CS responses across the Purkinje cell population (Yang and Lisberger, 2013; Yang and Lisberger, 2014). For the CSinfrequent Purkinje cells, the trial-over-trial learning in eye velocity is independent of the occurrence of a CS on the instruction trial (Fig. $8 E$ ). We suggest that the absence of correlations in their CS responses accounts for the absence of a link from the CS of the CS-infrequent Purkinje cells to the size of trial-over-trial behavioral learning.

Thus, CS-frequent and CS-infrequent Purkinje cells appear to differ in the reliability of their CS responses to the instruction, and in the absence of correlation among the CS responses of the CSinfrequent neurons. However, the basic CS-linked trial-over-trial plasticity is similar in the two groups. We suggest that CS-infrequent Purkinje cells show "wrong-way" learning in the ON-CS direction because synaptic potentiation on most trials dominates over the small amount of depression that can be mustered on the $20 \%$ of trials that show a CS response to the instruction.

CS-frequent versus CS-infrequent Purkinje cells: comparison with a prior sample population

It might make sense to stop after Figure 8, except that slight differences between the results in Figure 6 and the conclusions of 


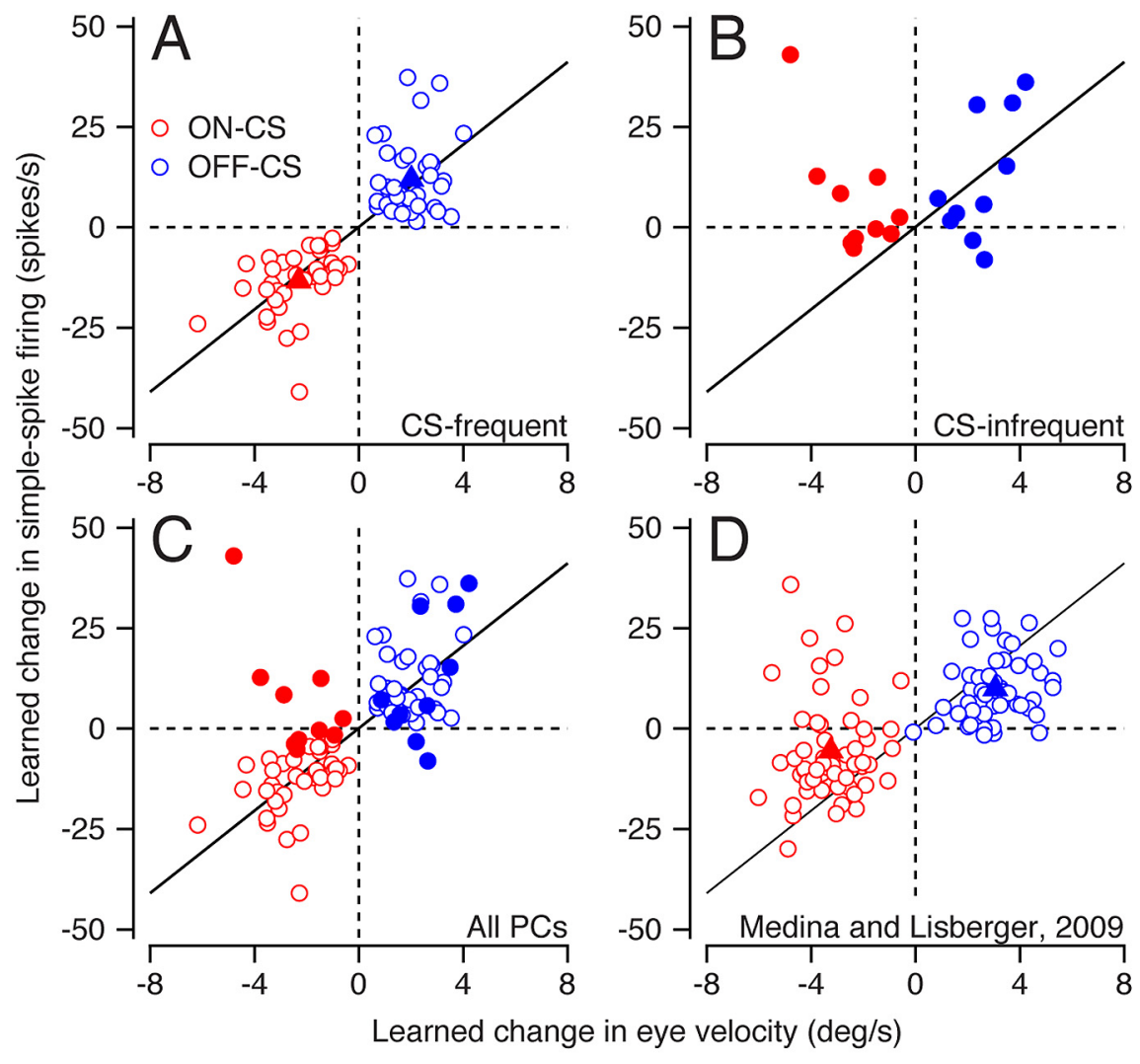

Figure 9. Comparison of asymptotic learning in CS-frequent and CS-infrequent Purkinje cells. Scatter plots show the learned change in simple-spike firing rate in the last 10 trials of a repeated-direction learning block versus the learned change in eye velocity in the same trials. Each symbol shows data for an individual neuron, red and blue symbols show data for the ON-CS and OFF-CS directions, and the same black line appears in each graph as the regression fit to the data in $\boldsymbol{A}$. $\mathbf{A}-\boldsymbol{C}$, Data from our sample; open and filled symbols show CS-frequent and CS-infrequent Purkinje cells. $\boldsymbol{D}$, Data from Purkinje cells used in Medina and Lisberger (2009). The filled red and blue triangles in $\boldsymbol{A}$ and $\boldsymbol{D}$ show the averages across the populations for ON-CS versus OFF-CS directions of learning.

a prior publication from our laboratory (Medina and Lisberger, 2009) require careful attention. The apparent, slight discrepancy lies in the comparison of the actual learned changes in simplespike firing rate and those predicted by the sensitivities to eye movement during baseline pursuit.

In our sample, we were able to use the probability of a CS response to the instruction to divide the Purkinje cells into two groups. We see differences in the general features of the learning in the two groups when we plot the learned change in simplespike firing in the last 10 trials of 100 consecutive learning trials with the same direction of instruction. The CS-frequent Purkinje cells (Fig. 9A) show positive or negative simple-spike learning for the same signs of behavioral learning, and plot around the line of slope 1. The CS-infrequent Purkinje cells plot around the line of slope 1 for the OFF-CS direction, but they show either very little or positive (wrong-way) simple-spike learning for the ON-CS direction.

In the sample of Medina and Lisberger (2008, 2009), few Purkinje cells had well isolated CS responses and it was not possible to divide the sample according to the probability of a $\mathrm{CS}$ response to the instruction. As a result, we have plotted the entire sample on the same graph (Fig. 9D). The overall distribution of their sample is similar to the overall distribution of ours (Fig. 9C). However, their sample showed a higher density of Purkinje cells with zero or positive (wrong-way) simplespike learning in the ON-CS direction. On this basis, we suggest that they recorded from a higher proportion of CS-infrequent Purkinje cells than we did.
We think that the apparent sampling difference accounts for a small discrepancy between our report and the previous one. In our report, the actual learned firing rate of the CS-frequent Purkinje cells exceeds that predicted firing rate from the sensitivity to eye movement during baseline pursuit for both the ON-CS and OFF-CS directions (Fig. 6A, C). In Figure 14 of Medina and Lisberger (2009), the actual and predicted firing rates were approximately equal. In their paper, the analysis combined Group 1 Purkinje cells with an apparent excess of Group 2 Purkinje cells, leading to smaller averages across the sample population of learned changes in firing rate (Fig. $9 D$, filled triangles). In contrast, our analysis included only identified CS-frequent Purkinje cells (Fig. 9A, filled triangles).

\section{Discussion}

We have recorded from Purkinje cells in the cerebellum during motor learning in the direction of smooth pursuit eye movements. Our experimental paradigms and analysis methods allow us to draw conclusions about what happens at multiple sites in the cerebellum during learning of a motor skill. We suggest that CS-linked trial-over-trial depression is a primary cause of trial-over-trial learning and also facilitates long-term learning. Other sites of plasticity would contribute over longer time courses and at later times.

\section{A primary role for CS-linked trial-over-trial plasticity in} the cerebellum

Our first conclusion is that CS-linked trial-over-trial depression of simple-spike firing in floccular Purkinje cells causes trial-overtrial learning in eye velocity. Two facts support this conclusion: (1) trial-over-trial learning and depression endure for the same duration: both are forgotten within $6 \mathrm{~s}$; (2) as described by Yang and Lisberger (2010), trial-over-trial learning shows a direction dependence that is related to the preferred directions for CS responses of the Purkinje cells in the floccular complex. Trial-overtrial learning is weak or nonexistent for downward instructions (Yang and Lisberger, 2010), in agreement with the paucity of Purkinje cells that have CS responses to downward instructions (Krauzlis and Lisberger, 1996). The disynaptic connection from floccular Purkinje cells to extraocular motoneurons (Highstein, 1973) also supports the causal link we propose.

Our second conclusion suggests a causal link to long-term learning from CS-linked trial-over-trial depression of simplespike responses and/or trial-over-trial learning. Again, two facts support this conclusion: (1) long instruction-test intervals do not support trial-over-trial depression and learning and lead to reduced long-term learning; and (2) the effect of instruction-test interval on long-term learning did not appear for downward instructive changes in target direction, in agreement with the absence of a large number of Purkinje cells that could undergo 
CS-linked trial-over-trial depression for downward instructions (Krauzlis and Lisberger, 1996).

We suggest that a neural mechanism related to trial-over-trial learning is a necessary prerequisite for one component of long-term learning in pursuit (Smith et al., 2006; Ethier et al., 2008). We favor the idea that CS-linked short-term depression of simple-spike firing is the obligate mechanism. However, trial-over-trial learning and long-term learning might be controlled in parallel by the same neural signals, without a causal link from trial-over-trial learning to long-term learning. In addition, some long-term learning occurs without any trial-over-trial effects. Longterm learning in the cerebellar cortex may be only partially dependent on CS-linked trial-over-trial depression, or plasticity might occur at other sites in the pursuit circuit.

We think of our experimental design as a strategy for deducing what happens in the cerebellum during motor learning. But, the effect of the instruction-test interval on long-term learning also raises a functional question. Under what circumstances do we repeat a movement frequently enough to take advantage of the extra learning power provided by short instruction-test intervals? Possible examples include the impressive results yielded by practice strategies, such as hitting a tennis ball against a backboard, or repeating a very brief line of piano music many times. Perhaps we should think of the extra learning that occurs with short intervals between movements as a clue for the best way to perfect a motor skill.

\section{Plasticity mechanisms at different sites expressed at different times during learning}

We interpret the progression of changes in simple-spike firing rate under different learning conditions as evidence that learning occurs initially at a subset of sites in the pursuit pathways and gets distributed across the circuit over time (Fig. 10). We suggest that the earliest learning results from CS-linked trial-over-trial depression of simple-spike firing rate, where the actual depression is $\sim 10$ times that predicted from the neurons' baseline relationship to the parameters of pursuit eye movement. We conclude that the Purkinje cells with ON-CS directions are carrying 10-times their normal burden in terms of driving eye movement. They may even be the sole site of trial-over-trial plasticity and may be solely responsible for the learned changes in eye velocity.

After the initial trial-over-trial changes, learning appears to progress partly through depression of the simple-spike responses in CS-frequent Purkinje cells that have CS responses for target motion in the direction of the instruction. Neural depression that exaggerates the behavioral learning in the CSfrequent Purkinje cells would compensate for the wrong-way changes in the CS-infrequent Purkinje cells, and the possible absence of learning in other pursuit pathways. Potentiation occurs in a variety of sites in the cerebellar circuits and is therefore another candidate mechanism for motor learning (Armano et al., 2000; Lev-Ram et al., 2002; Jörntell and Hansel, 2006; Belmeguenai et al., 2010). Indeed, our data suggest that learning involves potentiation in Purkinje cells whose ON-CS directions are opposite to the direction of the instruction. However, potentiation seems to evolve more slowly than depression in vivo and is somewhat weaker.
That the learned changes in simple-spike firing exaggerate the learned change in behavior after 100 repetitions of the same learning instruction suggests that Purkinje cells are shouldering a greater burden for driving learned eye velocity than for normal pursuit. This would be expected if some components of the pursuit circuit have not (yet) undergone plasticity. We predict that truly asymptotic learning would be distributed through the pursuit circuit so that every neuron's learned change in firing would match to its original baseline sensitivity to the eye movements of pursuit. It follows that (1) 100 learning trials creates an intermediate stage of learning, and (2) we would identify additional sites of plasticity and new components of learning if we followed pursuit learning for 2000, or maybe 20,000, trials. More distributed learning could arise through transfer of plasticity from the cerebellar cortex to the deep cerebellar nucleus (Miles and Lisberger, 1981; Zhang and Linden, 2006; Zheng and Raman, 2010), later learning in the cerebellar vermis (Suzuki and Keller, 1988; Takagi et al., 2000) or other brainstem areas, or engagement of other mechanisms of plasticity in the cerebellar cortex (Hansel et al., 2001; Carey, 2011).

\section{Participation in pursuit versus participation in learning}

One of the striking features of our data is that all Purkinje cells show CS-linked trial-over-trial depression of simple-spike firing rate. Yet, a small subset of CS-infrequent neurons nevertheless shows wrong-way changes in simple-spike responses after 100 learning trials in the ON-CS direction. We suggest that both trialover-trial depression and LTD are intact in CS-infrequent Purkinje cells, but that the number of CS responses is too small to support right-way learning. In addition, it appears that the CS responses of CS-infrequent Purkinje cells are not correlated with each other or with those in the larger group of CS-frequent Purkinje cells. Thus, the wrong-way learning in CS-infrequent Purkinje cells appears to be a consequence of the organization of their climbing fiber inputs, not of their intrinsic physiology.

Our data confirmed that the two groups of Purkinje cells show similar responses during baseline pursuit (Medina and Lisberger, 2008), raising the possibility that they work together to drive pursuit. However, they appear to work against each other during learning in the $\mathrm{ON}-\mathrm{CS}$ direction. As an alternative, it is possible that CS-infrequent Purkinje cells do not have output connections to the pursuit circuit even though they respond strongly during baseline pursuit. Our methods do not allow us to test this viable alternative. 


\section{Pursuit learning mechanisms in a broader context}

Others have provided evidence for multiple components of motor learning with different plasticity mechanisms, sites, and time courses. In the vestibulo-ocular reflex, plasticity occurs in both the cerebellar cortex and the deep cerebellar nucleus (Lisberger, 1994; Blazquez et al., 2006). There are two components of learning that do versus do not depend on climbing-fiber inputs (Ke et al., 2009). Learning occurs on multiple time courses (van Alphen and De Zeeuw, 2002). Vestibulo-ocular reflex learning to increase versus decrease the size of the reflex employs different cellular mechanisms of plasticity (Boyden et al., 2006). In eyelid conditioning, one popular hypothesis is that learning occurs first in the cerebellar cortex and then is transferred to the deep cerebellar nucleus (Ohyama et al., 2006). Only learned timing remains in the cerebellar cortex (Kalmbach et al., 2010). In learning for arm movements and saccadic eye movements, there are multiple sites of learning (Ethier et al., 2008) and a diversity of time courses (Smith et al., 2006).

Our paper goes beyond the prior papers by showing how different parts of a circuit and different mechanisms of plasticity participate in motor learning over different time courses. We show that the climbing-fiber input plays a special, primary role in pursuit learning. We also demonstrate a critical relationship between short-term and long-term learning. We suggest that the primary site of learning is in Purkinje cells that show frequent CS responses for the instruction direction. These Purkinje cells undergo learning from the very first learning trial, and seem to employ two important mechanisms of plasticity: short-term and long-term CS-linked synaptic depression. We suggest that learning occurs more slowly in Purkinje cells with CS responses that prefer the opposite direction relative to the instruction, and that the mechanism of plasticity for them involves synaptic potentiation. Still later learning may occur at a variety of sites. Our suggestions emphasize the systems nature of motor learning and the likely participation of multiple forms of plasticity at different sites and different times.

\section{References}

Albus JS (1971) A theory of cerebellar function. Math Biosci 10:25-61. CrossRef

Armano S, Rossi P, Taglietti V, D'Angelo E (2000) Long-term potentiation of intrinsic excitability at the mossy fiber-granule cell synapse of rat cerebellum. J Neurosci 20:5208-5216. Medline

Belmeguenai A, Hosy E, Bengtsson F, Pedroarena CM, Piochon C, Teuling E, He Q, Ohtsuki G, De Jeu MT, Elgersma Y, De Zeeuw CI, Jörntell H, Hansel C (2010) Intrinsic plasticity complements long-term potentiation in parallel fiber input gain control in cerebellar Purkinje cells. J Neurosci 30:13630-13643. CrossRef Medline

Blazquez PM, Hirata Y, Highstein SM (2004) The vestibulo-ocular reflex as a model system for motor learning: what is the role of the cerebellum? Cerebellum 3:188-192. CrossRef Medline

Blazquez PM, Hirata Y, Highstein SM (2006) Chronic changes in inputs to dorsal Y neurons accompany VOR motor learning. J Neurophysiol 95: 1812-1825. CrossRef Medline

Boyden ES, Katoh A, Raymond JL (2004) Cerebellum-dependent learning: the role of multiple plasticity mechanisms. Annu Rev Neurosci 27:581609. CrossRef Medline

Boyden ES, Katoh A, Pyle JL, Chatila TA, Tsien RW, Raymond JL (2006) Selective engagement of plasticity mechanisms for motor memory storage. Neuron 21:823-834. Medline

Carey MR (2011) Synaptic mechanisms of sensorimotor learning in the cerebellum. Curr Opin Neurobiol 21:609-615. CrossRef Medline

Christian KM, Thompson RF (2003) Neural substrates of eye blink conditioning: acquisition and retention. Learn Mem 10:427-455. CrossRef Medline

D’Angelo E, Rossi P, Armano S, Taglietti V (1999) Evidence for NMDA and
mGlu receptor-dependent long-term potentiation of mossy fiber-granule cell transmission in rat cerebellum. J Neurophysiol 81:277-287. Medline

Ethier V, Zee DS, Shadmehr R (2008) Spontaneous recovery of motor memory during saccade adaptation. J Neurophysiol 99:2577-2583. CrossRef Medline

Gao Z, van Beugen BJ, De Zeeuw CI (2012) Distributed synergistic plasticity and cerebellar learning. Nat Rev Neurosci 13:619-635. CrossRef Medline

Gilbert PF, Thach WT (1977) Purkinje cell activity during motor learning. Brain Res 128:309-328. CrossRef Medline

Hansel C, Linden DJ, D'Angelo E (2001) Beyond parallel fiber LTD: the diversity of synaptic and non-synaptic plasticity in the cerebellum. Nat Neurosci 4:467-475. Medline

Highstein SM (1973) Synaptic linkage in the vestibulo-ocular and cerebellovestibular pathways to the VIth nucleus in the rabbit. Exp Brain Res 17:301-314. Medline

Ito M (1972) Neural design of the cerebellar motor control system. Brain Res 40:81-84. CrossRef Medline

Jörntell H, Ekerot CF (2002) Reciprocal bidirectional plasticity of parallel fiber receptive fields in cerebellar Purkinje cells and their afferent interneurons. Neuron 34:797-806. CrossRef Medline

Jörntell H, Ekerot CF (2003) Receptive field plasticity profoundly alters the cutaneous parallel fiber synaptic input to cerebellar interneurons in vivo. J Neurosci 23:9620-9631. Medline

Jörntell H, Hansel C (2006) Synaptic memories upside down: bidirectional plasticity at cerebellar parallel fiber-Purkinje cell synapses. Neuron 52: 227-238. CrossRef Medline

Kahlon M, Lisberger SG (1996) Coordinate system for learning in the smooth pursuit eye movements of monkeys. J Neurosci 16:7270-7283. Medline

Kahlon M, Lisberger SG (2000) Changes in the responses of Purkinje cells in the floccular complex of monkeys after motor learning in smooth pursuit eye movements. J Neurophysiol 84:2945-2960. Medline

Kalmbach BE, Davis T, Ohyama T, Riusech F, Nores WL, Mauk MD (2010) Cerebellar cortex contributions to the expression and timing of conditioned eyelid responses. J Neurophysiol 103:2039-2049. CrossRef Medline

Ke MC, Guo CC, Raymond JL (2009) Elimination of climbing fiber instructive signals during motor learning. Nat Neurosci 12:1171-1179. CrossRef Medline

Krauzlis RJ, Lisberger SG (1996) Directional organization of eye movement and visual signals in the floccular lobe of the monkey cerebellum. Exp Brain Res 109:289-302. Medline

Lev-Ram V, Wong ST, Storm DR, Tsien RY (2002) A new form of cerebellar long-term potentiation is postsynaptic and depends on nitric oxide but not cAMP. Proc Natl Acad Sci U S A 99:8389-8393. CrossRef Medline

Lisberger SG (1994) Neural basis for motor learning in the vestibuloocular reflex of primates. III. Computational and behavioral analysis of the sites of learning. J Neurophysiol 72:974-998. Medline

Lisberger SG (2009) Internal models of eye movement in the floccular complex of the monkey cerebellum. Neuroscience 162:763-776. CrossRef Medline

Lisberger SG, Fuchs AF (1978) Role of primate flocculus during rapid behavioral modification of vestibuloocular reflex. I. Purkinje cell activity during visually guided horizontal smooth-pursuit eye movements and passive head rotation. J Neurophysiol 41:733-763. Medline

Lisberger SG, Pavelko TA (1986) Vestibular signals carried by pathways subserving plasticity of the vestibulo-ocular reflex in monkeys. J Neurosci 6:346-354. Medline

Lisberger SG, Pavelko TA, Broussard DM (1994) Neural basis for motor learning in the vestibulo-ocular reflex of primates: I. Changes in the responses of brain stem neurons that receive monosynaptic inhibition from the flocculus and ventral paraflocculus. J Neurophysiol 72:928-953. Medline

Maekawa K, Simpson JI (1973) Climbing fiber responses evoked in vestibulecerebellum of rabbit from visual system. J Neurophysiol 36:649-666. Medline

Marr D (1969) A theory of cerebellar cortex. J Physiol (Lond) 202:437-470.

Maruta J, Hensbroek RA, Simpson JI (2007) Intraburst and interburst signaling by climbing fibers. J Neurosci 27:11263-11270. CrossRef Medline

Medina JF, Lisberger SG (2008) Links from complex spikes to local plasticity and motor learning in the cerebellum of awake-behaving monkeys. Nat Neurosci 11:1185-1192. CrossRef Medline

Medina JF, Lisberger SG (2009) Encoding and decoding of learned smooth- 
pursuit eye movements in the floccular complex of the monkey cerebellum. J Neurophysiol 102:2039-2054. CrossRef Medline

Medina JF, Mauk MD (2000) Computer simulation of cerebellar information processing. Nat Neurosci 3:1205-1211. CrossRef Medline

Medina JF, Nores WL, Ohyama T, Mauk MD (2000) Mechanisms of cerebellar learning suggested by eyelid conditioning. Curr Opin Neurobiol 10:717-724. CrossRef Medline

Medina JF, Carey MR, Lisberger SG (2005) The representation of time for motor learning. Neuron 45:157-167. CrossRef Medline

Miles FA, Fuller JH (1975) Visual tracking and the primate flocculus. Science 189:1000-1002. Medline

Miles FA, Lisberger SG (1981) Plasticity in the vestibulo-ocular reflex: a new hypothesis. Annu Rev Neurosci 4:273-299. CrossRef Medline

Nelson AB, Gittis AH, du Lac S (2005) Decreases in CaMKII activity trigger persistent potentiation of intrinsic excitability in spontaneously firing vestibular nucleus neurons. Neuron 46:623-631. CrossRef Medline

Ohyama T, Nores WL, Medina JF, Riusech FA, Mauk MD (2006) Learninginduced plasticity in deep cerebellar nucleus. J Neurosci 26:12656-12663. CrossRef Medline

Ramachandran R, Lisberger SG (2005) Normal performance and expression of learning in the vestibulo-ocular reflex (VOR) at high frequencies. J Neurophysiol 93:2028-2038. Medline

Rambold H, Churchland A, Selig Y, Jasmin L, Lisberger SG (2002) Partial ablations of the flocculus and ventral paraflocculus in monkeys cause linked deficits in smooth pursuit eye movements and adaptive modification of the VOR. J Neurophysiol 87:912-924. Medline

Rashbass C (1961) The relationship between saccadic and smooth tracking eye movements. J Physiol 159:326-338. Medline

Smith MA, Ghazizadeh A, Shadmehr R (2006) Interacting adaptive processes with different timescales underlie short-term motor learning. PLoS Biol 4:e179. Medline

Stone LS, Lisberger SG (1990a) Visual responses of Purkinje cells in the cerebellar flocculus during smooth-pursuit eye movements in monkeys. I. Simple-spikes. J Neurophysiol 63:1241-1261. Medline
Stone LS, Lisberger SG (1990b) Visual responses of Purkinje cells in the cerebellar flocculus during smooth-pursuit eye movements in monkeys. II. Complex spikes. J Neurophysiol 63:1262-1275. Medline

Suzuki DA, Keller EL (1988) The role of the posterior vermis of monkey cerebellum in smooth-pursuit eye movement control. II. Target velocityrelated Purkinje cell activity. J Neurophysiol 59:19-40. Medline

Takagi M, Zee DS, Tamargo RJ (2000) Effects of lesions of the oculomotor cerebellar vermis on eye movements in primate: smooth pursuit. J Neurophysiol 83:2047-2062. Medline

Thompson RF (2013) An essential memory trace found. Behav Neurosci 127:669-675. CrossRef Medline

van Alphen AM, De Zeeuw CI (2002) Cerebellar LTD facilitates but is not essential for long-term adaptation of the vestibulo-ocular reflex. Eur J Neurosci 16:486-490. CrossRef Medline

Wulff P, Schonewille M, Renzi M, Viltono L, Sassoè-Pognetto M, Badura A, Gao Z, Hoebeek FE, van Dorp S, Wisden W, Farrant M, De Zeeuw CI (2009) Synaptic inhibition of Purkinje cells mediates consolidation of vestibulo-cerebellar motor learning. Nat Neurosci 12:1042-1049. CrossRef Medline

Yang Y, Lisberger SG (2010) Learning on multiple timescales in smooth pursuit eye movements. J Neurophysiol 104:2850-2862. CrossRef Medline

Yang Y, Lisberger SG (2013) Interaction of plasticity and circuit organization during the acquisition of cerebellum-dependent motor learning. eLife 2:e01574. CrossRef Medline

Yang Y, Lisberger SG (2014) Purkinje-cell plasticity and cerebellar motor learning are graded by complex-spike duration. Advance online publication. doi:10.1038/nature13282. CrossRef

Zee DS, Yamazaki A, Butler PH, Gücer G (1981) Effects of ablation of flocculus and paraflocculus of eye movements in primate. J Neurophysiol 46:878-899. Medline

Zhang W, Linden DJ (2006) Long-term depression at the mossy fiber-deep cerebellar nucleus synapse. J Neurosci 26:6935-6944. CrossRef Medline

Zheng N, Raman IM (2010) Synaptic inhibition, excitation, and plasticity in neurons of the cerebellar nuclei. Cerebellum 9:56-66. CrossRef Medline 